\title{
Life course path analysis of total and central adiposity throughout adolescence on adult blood pressure and insulin resistance
}

\author{
J. Araújo a,b,*, M. Severo ${ }^{\mathrm{a}, \mathrm{b}}$, S. Santos a, E. Ramos ${ }^{\mathrm{a}, \mathrm{b}}$ \\ ${ }^{a}$ EPIUnit - Institute of Public Health, University of Porto, Porto, Portugal \\ ${ }^{b}$ Department of Clinical Epidemiology, Predictive Medicine and Public Health, University of Porto Medical School, Porto, Portugal \\ Received 9 June 2016; received in revised form 14 December 2016; accepted 19 December 2016 \\ Available online 24 December 2016
}

\section{KEYWORDS \\ Life course; \\ Path analysis; \\ Adolescence; \\ Adiposity; \\ Blood pressure; \\ Insulin resistance}

\begin{abstract}
Background and aim: We aimed to study whether the effect of adolescent adiposity on adult blood pressure and insulin resistance was mediated by adult adiposity.

Methods and results: Data from the EPITeen cohort at 13, 17 and 21 years was used $(\mathrm{n}=2211)$. Sex- and age-specific body mass index z-scores (BMIz) and waist-to-hip ratio (WHR) were used as indicators of total and central adiposity, respectively. Systolic blood pressure (SBP), glucose and insulin were assessed at 21 years and the homeostasis model assessment (HOMA-IR) was used as a marker of insulin resistance. Path analysis was applied to evaluate direct and indirect effects of adiposity $(13,17$ and 21y) on adult SBP and HOMA-IR, separately for total and central adiposity and for each outcome. Results are presented as standardized regression coefficients [ $\beta$ $(95 \% \mathrm{CI})]$. The total effect of BMIz at 13 years on SBP at 21 years was $0.211(0.178 ; 0.244)$, totally mediated by adult BMIz. Total effect of BMIz 13y on HOMA-IR was $0.248(0.196 ; 0.299)$. Although this effect was mostly mediated by BMIz 21y, an additional direct effect from BMIz 17y was found $[\beta=-0.240(-0.315 ;-0.164)]$. Central adiposity was also positively associated with SBP and HOMA-IR at 21 years, and the effect of adolescent WHR was totally mediated by adult WHR for both outcomes.

Conclusions: The effect of adolescent adiposity on adult SBP and HOMA-IR was mostly mediated by adult adiposity. However, for HOMA-IR an additional direct effect from total adiposity at 17 years was found.

(c) 2017 The Italian Society of Diabetology, the Italian Society for the Study of Atherosclerosis, the Italian Society of Human Nutrition, and the Department of Clinical Medicine and Surgery, Federico II University. Published by Elsevier B.V. All rights reserved.
\end{abstract}

\section{Introduction}

The childhood obesity epidemic experienced in last decades, especially in developed countries, is likely to introduce changes in the pattern and timing of cardiovascular disease (CVD), leading to an epidemic of early onset of CVD risk factors and events [1]. Studies have shown that changes in adiposity in specific periods of the

\footnotetext{
* Corresponding author. Instituto de Saúde Pública, Rua das Taipas 135, 4050-600 Porto, Portugal. Fax: +351 222061821.

E-mail address: joana.araujo@ispup.up.pt (J. Araújo).
}

paediatric age are influential for future cardio metabolic health [2-6]. However, due to the expected tracking of adiposity from childhood to adulthood [7], there is increasing interest in decomposing direct effects of early adiposity on adult outcomes, from those mediated by adult adiposity. However, most studies measuring the independent effects of adiposity in paediatric age on adult outcomes used standard regression techniques to adjust for adult adiposity $[2-4,8,9]$, which has been suggested to cause statistical artefacts and to be controversial regarding the interpretation of the results [10,11]. For example, in these models the adjustment for adult adiposity controls for change in adiposity between measurements, not only 
for adult body size [11], and collinearity problems may also be present [10]. Path analysis has been suggested as a superior statistical method in comparison to other standard regressions, since allows the estimation of direct and indirect effects, producing easily interpretable results [6]. Additionally, it may deal more efficiently with collinearity [6], although some authors argue that it can still be a problem in life course path analysis [12].

The effects of adiposity on cardiovascular risk factors may emerge at different periods over the life course depending on the outcomes studied. Obesity is associated with both insulin resistance and blood pressure, and these outcomes may have some shared mechanisms, such as obesity-induced inflammation [13]. However, in youth insulin resistance or type 2 diabetes are more common consequences of obesity than hypertension [14,15]. Studies have also suggested that insulin resistance is implicated in the subsequent development of hypertension [16]. Still, the evidence is limited and controversial.

In adults, abdominal fat, particularly visceral fat, has been clearly associated with the highest risk of CVD disease [17]. However, in paediatric age studies addressing changes in adiposity have mainly focused on total adiposity, remaining the effect of central adiposity under investigated.

Therefore, we extended the evidence of previous studies on the effect of adolescent adiposity on adult CVD risk factors, by studying both total and central adiposity, and applying life course path analysis. Specifically, our aim was to study whether the effect of adolescent adiposity on adult blood pressure and insulin resistance is mediated by adult adiposity.

\section{Methods}

\section{Study sample}

Data was collected as part of the EPITeen study (Epidemiological Health Investigation of Teenagers in Porto), a population-based cohort that recruited 13-year-old adolescents born in 1990 and enrolled at public and private schools of Porto, Portugal, during 2003/2004 [18]. A second evaluation took place in 2007/2008 and a third evaluation in 2011-2013, when participants were on average 17 and 21 years, respectively.

Our study complies with the Declaration of Helsinki and the Ethic Committee of Hospital S. João and the Ethic Committee of the Institute of Public Health from the University of Porto approved the research protocol. Written informed consent was obtained from parents and adolescents in the first and second study waves, and from participants in the third study wave.

At the recruitment, 2786 eligible participants were identified and 2159 (77.5\%) agreed to participate. In the second wave, 1716 participants (79.5\%) were re-evaluated, and a new group of 783 adolescents were evaluated for the first time and integrated the cohort as they moved to the schools of Porto. In the third study wave, 1764 participants were re-evaluated. From the entire cohort $(n=2942)$,
2211 (75\%) participants with anthropometric data in, at least, two study waves were included in this analysis.

\section{Measures}

\section{Anthropometrics}

Weight and height were obtained with the subject in light indoor clothes and no shoes. Weight was measured in kilograms, to the nearest tenth, using a digital scale and height was measure in centimetres, to the nearest tenth, according to standardized procedures. Waist and hip circumferences were measured to the nearest $0.1 \mathrm{~cm}$ with a flexible and non-distensible tape, avoiding exertion of pressure on the tissues and with the subject standing. Waist circumference was measured midway between the lower limit of the rib cage and the iliac crest, at the end of gentle expiration, and hip circumference was measured on the maximum circumference over the femoral trochanters.

As a measure of total adiposity we used BMI, standardized according to the sex- and age-specific growth reference data for 5-19 years, from the World Health Organization (WHO) [19]. BMI from the third study wave at 21 years was standardized using the WHO z-scores for 19 years. The waist-to-hip ratio was used as indicator of central adiposity.

\section{Cardio metabolic parameters at 21 years}

An overnight fast intravenous blood sample was taken from an antecubital vein. Glucose was measured using conventional methods with an Olympus AU5400 ${ }^{\circledR}$ automated clinical chemistry analyzer (Beckman-Coulter ${ }^{\circledR}$ ). Insulin was measured by electro chemiluminescent immunoassay using a Cobas ${ }^{\circledR}$ e411 automated analyzer $\left(\right.$ Roche $\left.^{\circledR}\right)$. All determinations took place in the Clinical Pathology Department of the São João Hospital Center, Porto. The homeostasis model method (HOMA-IR) was used as a marker of insulin resistance, based on fasting glucose and insulin concentrations: HOMA-IR = insulin $(\mu \mathrm{U} / \mathrm{ml}) *$ glucose $(\mathrm{mg} / \mathrm{dl}) / 405[20]$.

Blood pressure was measured using an oscillometric method (OMRON Blood Pressure Monitor, M6 Comfort), according to standardized procedures. After $10 \mathrm{~min}$ of rest, two blood pressure measurements were taken, separately by at least five minutes; a third measure was taken if the difference between the first two was higher than $5 \mathrm{mmHg}$. The average of the two closest measurements was used in the analysis.

\section{Statistical analysis}

Data are presented as counts and percentages or mean and standard deviation (SD), for categorical and quantitative variables, respectively.

Path analysis was used to evaluate the independent associations between BMIz and WHR at each age $(13,17$ and 21 years) with adult (21 years) SBP or HOMA-IR. Results are presented as standardized regression coefficients $(\beta)$ and $95 \%$ confidence intervals $(95 \% \mathrm{CI})$ to allow direct comparisons between different exposures and outcomes. 
Based on previous studies suggesting that the effect of adolescent adiposity on adult outcomes is mostly mediated by adult adiposity, we started the analysis with a reduced model as illustrated in Fig. 1. This model included only the associations between BMIz/WHR at subsequent ages (13-17 years, and 17-21 years) and the association between BMIz/WHR at 21 years and the outcome at 21 years (SBP/HOMA-IR). In the second step, we added additional pathways (one by one), according to the model modification indices. Additional associations were added only if modification index was over $6.63\left(\chi_{0.990 ;(1)}^{2}\right)$ to minimize the probability of type I error. The final models included the direct pathway of BMIz/WHR between 13 and 21 years, and specifically in the model for BMIz and HOMA-IR, the direct pathway from BMIz 17y and HOMA-IR $21 \mathrm{y}$.

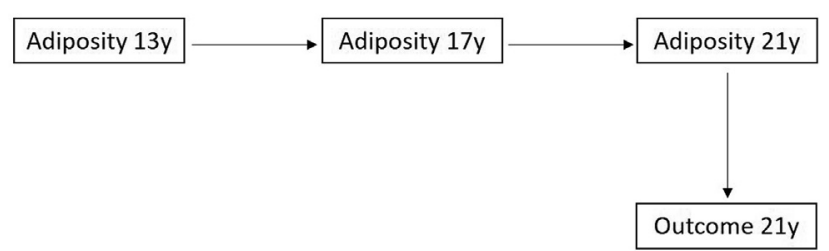

Figure 1 Illustrative example of the reduced path analysis model for the association between adiposity throughout adolescence and the outcome in adulthood.

The goodness-of-fit of the path analysis model was evaluated through three fit indexes of confirmatory factor analysis: the root-mean-square error of approximation (RMSEA), Bentler comparative fit index (CFI) and TuckerLewis non-normed fit index (TLI). Values of RMSEA $<0.06$, and CFI and TLI $>0.95$ indicate good model fit [21]. Indirect effects were calculated as the product of two (or more) regression coefficients, which are equal to the difference between the total and direct effects. One limitation of this method is the assumption of normality distribution of standard errors, therefore we calculated confidence intervals based on 1000 Bootstrap sample replicates.

Path analyses were run in MPlus, version 5.2.

\section{Results}

Descriptive data on adiposity and cardio metabolic parameters are presented in Table 1. In general, weight, BMI and waist circumference mean levels increased with age, as expected. In comparison to females, blood pressure and glucose mean levels were higher in males, while insulin and HOMA-IR were lower, but within normal ranges in both sexes.

Pearson correlations between adiposity measures in the three study waves and SBP and HOMA-IR at 21 years are presented in Table S1, Supplementary material 1.

Direct effects estimated from the path analysis are shown in Figs. 2 and 3, and total and indirect effects are presented in Table 2. The association between BMIz in adolescence (13 and 17 years) and SBP in adulthood was
Table 1 Adiposity and cardio metabolic parameters of the study sample, by sex.

\begin{tabular}{llll}
\hline & & \multicolumn{1}{c}{ Females } & \multicolumn{1}{c}{ Males } \\
\hline Adiposity parameters & & \multicolumn{2}{c}{ Mean (SD) } \\
Weight $(\mathrm{kg})$ & 13 years & $52.6(9.8)$ & $54.7(12.0)$ \\
& 17 years & $57.3(9.2)$ & $67.6(11.4)$ \\
& 21 years & $59.0(10.7)$ & $72.6(12.4)$ \\
BMI $\left(\mathrm{Kg} / \mathrm{m}^{2}\right)$ & 13 years & $21.0(3.4)$ & $20.6(3.6)$ \\
& 17 years & $22.0(3.2)$ & $22.4(3.4)$ \\
& 21 years & $22.6(4.0)$ & $23.6(3.7)$ \\
BMIz & 13 years & $0.40(0.99)$ & $0.47(1.16)$ \\
& 17 years & $0.22(0.90)$ & $0.29(1.05)$ \\
WC (cm) & 21 years & $0.20(1.04)$ & $0.31(1.10)$ \\
& 13 years & $71.5(7.9)$ & $73.2(9.3)$ \\
WHR & 17 years & $74.2(7.9)$ & $78.2(8.6)$ \\
& 21 years & $73.8(9.3)$ & $81.7(9.3)$ \\
13 years & $0.79(0.06)$ & $0.83(0.06)$ \\
Cardio metabolic parameters $21 y$ & \multicolumn{2}{c}{ Mean (SD) } \\
SBP (mmHg) & & $102.0(8.7)$ & $115.9(10.4)$ \\
DBP (mmHg) & & $68.1(7.4)$ & $69.6(7.5)$ \\
Glucose (mg/dl) & & $81.1(8.2)$ & $85.4(7.6)$ \\
Insulin (mg/dl) & & $9.5(4.9)$ & $8.2(4.7)$ \\
HOMA-IR & & $1.91(1.06)$ & $1.76(1.10)$ \\
\hline
\end{tabular}

BMIz: body mass index z-score; WHR: waist-to-hip ratio; SBP: systolic blood pressure; DBP: diastolic blood pressure; HOMA-IR: insulin resistance index.

totally mediated by adiposity tracking (Fig. 2A). The total effect $[\beta(95 \% \mathrm{CI})]$ of BMIz at 13 years on SBP at 21 years was $0.211(0.178 ; 0.244)$; majority (79\%) of it was explained by the pathway BMIz 13y, BMIz 17y, BMIz 21y to SBP 21y [0.166 (0.136; 0.195)], while $21 \%$ of the effect occurred through the pathway from BMIz 13y directly to BMIz 21y, to SBP 21y (Table 2). Total effect of BMIz 13y on SBP 21y and the effect of BMIz 17y were weaker than the effect of BMIz 21y on SBP 21y [ $\beta=0.284(0.241 ; 0.327)]$. The associations of BMIz with HOMA-IR were stronger than with SBP (Fig. 2B and Table 2). In general, the pathways for HOMA-IR were similar to those found for SBP, with the effect of BMIz 13 years being mostly mediated by adult BMIz. However, an additional direct effect between BMIz 17y and HOMA-IR 21y was found (Fig. 2B). The total and positive effect of BMIz 17y was 0.179 (0.114; 0.244), which is the sum of the negative direct effect on the outcome $[\beta=-0.240(-0.315 ;-0.164)]$ and the positive indirect effect through BMIz 21y [ $\beta=0.418(0.350 ; 0.487)]$ (Table 2). The coefficient of the direct effect of BMIz 17y is adjusted for BMIz 21 years, and therefore it should be interpreted as the change on HOMA-IR for one unit increase in the change (BMIz 17y - BMIz 21y); one unit increase in this variable corresponds to the decrease of one SD of the BMIz from 17 to 21 years and it is associated with lower HOMA-IR at 21 years. Regarding central adiposity, the effect of adolescent WHR was totally mediated by adult WHR for both SBP and HOMA-IR at 21 years (Fig. 3). The total effect of WHR 13y on SBP 21y was 0.219 (0.166; $0.271)$, and the direct effect from WHR $21 \mathrm{y}$ was 0.444 $(0.410 ; 0.478)$. The total effect of WHR 13y on HOMA-IR 


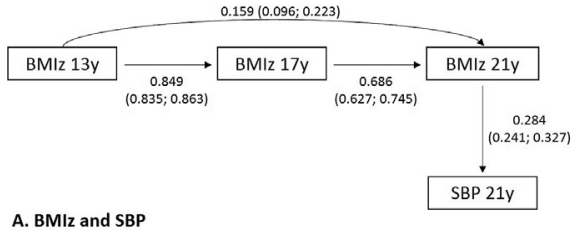

$X^{2}(2)=4.20, p=0.122 ;$ RMSEA index $=0.022 ; \mathrm{CFI}=0.999 ; \mathrm{TL}=0.998$

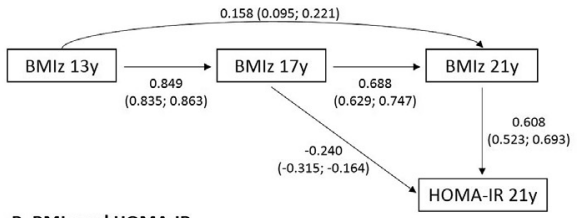

B. BMIz and HOMA-IR

$X^{2}(1)=2.06, p=0.151 ;$ RMSEA index $=0.022 ; \mathrm{CFI}=1.000 ; \mathrm{TL}=0.999$

Figure 2 Path analysis diagram for the regression of BMIz on SBP (A) and HOMA-IR (B) at 21 years. Data are standardized regression coefficients and 95\% confidence intervals. BMIz: body mass index z-score; SBP: systolic blood pressure; HOMA-IR: insulin resistance index; RMSEA: root-meansquare error of approximation; CFI: comparative fit index; TLI: Tucker-Lewis non-normed fit index.

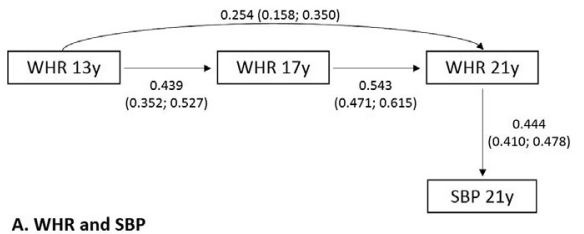

$X^{2}(2)=1.50, p=0.473 ;$ RMSEA index $=0.000 ; \mathrm{CFI}=1.000 ; \mathrm{TL}=1.001$

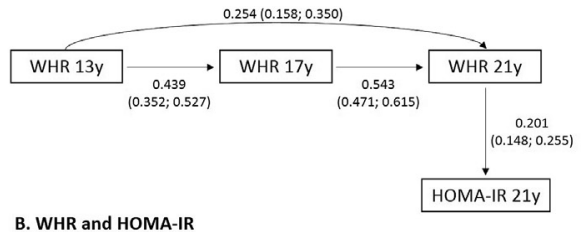

$X^{2}(2)=1.19, p=0.552 ;$ RMSEA index $=0.000 ; \mathrm{CF}=1.000 ; \mathrm{TL}=1.002$.

Figure 3 Path analysis diagram for the regression of WHR on SBP (A) and HOMA-IR (B) at 21 years. Data are standardized regression coefficients and 95\% confidence intervals. WHR: waist-to-hip ratio; SBP: systolic blood pressure; HOMA-IR: insulin resistance index; RMSEA: root-mean-square error of approximation; CFI: comparative fit index; TLI: Tucker-Lewis non-normed fit index.

$21 \mathrm{y}$ was $0.099(0.063 ; 0.135)$, and the direct effect from WHR 21y was $0.201(0.148 ; 0.255)$.

\section{Discussion}

Our study showed that the effect of adolescent total adiposity on adult blood pressure and insulin resistance was mostly mediated by adult adiposity, although results suggest that total adiposity throughout adolescence also influences adult insulin resistance, independently of adult adiposity. Our results also showed that central adiposity was associated with insulin resistance and blood pressure, but the effect was completely mediated by adult central adiposity.

\section{Methodological considerations}

More accurate and precise measurements of body composition are difficult to implement in large population-based studies, therefore, we used simple anthropometric indicators of adiposity. Although BMI cannot distinguish fat mass from fat free mass, it correlates well with total body mass [22]. However, its use in paediatric age raises additional questions related to the comparability of this measure across childhood and adolescence, and therefore we computed the BMI z-scores according to the WHO growth reference. This is particularly important in our study, since we analysed the effect of adiposity at different ages during a period of growth, and this approach minimizes misclassification differences between ages.

Although waist circumference has been frequently used as an indicator of central adiposity, it is acknowledged above all as a surrogate of total adiposity that is also influenced by abdominal adiposity [23]. Therefore, WC may be a valuable indicator of central fat, only when corrected for total adiposity. In our study, we used the WHR as an indicator of central adiposity. This ratio has been recognized as a measure of body fat distribution, representing abdominal fat [24], and it has been shown to correlate well with cardiovascular risk factors $[25,26]$.

The strengths of our study include its population-based nature and the prospective design. The young age of our participants also minimizes potential effects of reverse causation due to complications of disease or medications; prevalence of self-reported type 1 or type 2 diabetes in our sample at 21 years was $0.4 \%$ and hypertension was $0.1 \%$. Although our final results were computed in the subsample of participants presenting at least two adiposity measurements, a complete case analysis in 1172 participants retrieved similar results, suggesting that no major selection bias exists. Our results were based on path analysis, which allowed the calculation of total, direct and indirect effects of adiposity at different ages, on cardio metabolic outcomes in adulthood. It allowed an easier interpretation of the life course adiposity effects on adult outcomes, overcoming the problems of adjusting for adult attained adiposity [6].

Blood pressure was measured using an automatic device, rather than with a mercury sphygmomanometer. However, oscillometric devices such as the OMRON M6 Comfort used in our study have been shown to be valid for blood pressure assessment [27], even in obese adults [28].

Path analysis models did not include any confounder variables, and we could not exclude that our estimates would be attenuated if other factors were taken into account. However, as we were interested in measuring the effects of adiposity at different ages, those effects are expected to be influenced by similar confounder factors across age. 
Table 2 Total and indirect effects derived from the path analysis model for the association between adiposity (BMIz and WHR) and outcomes (SBP and HOMA-IR) at 21 years.

\begin{tabular}{|c|c|c|}
\hline \multirow[b]{2}{*}{ BMIz } & \multirow{2}{*}{$\frac{\text { Standardized } \beta(95 \% \mathrm{CI})}{\text { SBP 21y }}$} & \multirow{2}{*}{$\frac{\text { Standardized } \beta(95 \% \mathrm{CI})}{\text { HOMA-IR 21y }}$} \\
\hline & & \\
\hline Total effect of BMIz 13y & $0.211(0.178 ; 0.244)$ & $0.248(0.196 ; 0.299)$ \\
\hline \multicolumn{3}{|l|}{ Indirect effects } \\
\hline BMIz 13y $\rightarrow$ BMIz 17y $\rightarrow$ BMIz 21y & $0.166(0.136 ; 0.195)$ & $0.355(0.296 ; 0.414)$ \\
\hline BMIz 13y $\rightarrow$ BMIz 17y & - & $-0.203(-0.267 ;-0.140)$ \\
\hline BMIz 13y $\rightarrow$ BMIz 21y & $0.045(0.026 ; 0.065)$ & $0.096(0.055 ; 0.137)$ \\
\hline Total effect of BMIz 17y & $0.195(0.161 ; 0.229)$ & $0.179(0.114 ; 0.244)$ \\
\hline \multicolumn{3}{|l|}{ Indirect effects } \\
\hline BMIz 17y $\rightarrow$ BMIz 21y & $0.195(0.161 ; 0.229)$ & $0.418(0.350 ; 0.487)$ \\
\hline WHR & SBP 21y & HOMA-IR 21y \\
\hline Total effect of WHR 13y & $0.219(0.166 ; 0.271)$ & $0.099(0.063 ; 0.135)$ \\
\hline \multicolumn{3}{|l|}{ Indirect effects } \\
\hline WHR 13y $\rightarrow$ WHR 17y $\rightarrow$ WHR 21y & $0.106(0.087 ; 0.125)$ & $0.048(0.033 ; 0.063)$ \\
\hline WHR $13 \mathrm{y} \rightarrow$ WHR 21y & $0.113(0.068 ; 0.157)$ & $0.051(0.026 ; 0.076)$ \\
\hline Total effect of WHR 17y & $0.241(0.204 ; 0.278)$ & $0.109(0.077 ; 0.142)$ \\
\hline \multicolumn{3}{|l|}{ Indirect effects } \\
\hline WHR 17y $\rightarrow$ WHR 21y & $0.241(0.204 ; 0.278)$ & $0.109(0.077 ; 0.142)$ \\
\hline
\end{tabular}

\section{Interpretation of findings}

Our results suggest a stronger association of total adiposity with insulin resistance than with blood pressure, and support that adolescent total adiposity levels can affect future insulin resistance independently of adiposity at early adulthood. This result is not directly comparable with other studies since no data based on path analysis for insulin-resistance was previously reported. However, available evidence also suggests that there is an additional effect of BMI gains across the life span on adult insulin resistance, independently of the attained adult BMI $[2,3]$. Another study, however, found that adolescent BMI was not associated with risk of diabetes in adulthood, after adjustment for adult BMI, in contrast to the association found for coronary heart disease [29].

In our study the effect of BMIz in adolescence (at 13 or at 17 years) on adult SBP was totally mediated by adult BMIz. Our results are in accordance with those computed by path analysis in a sample of Danish men, showing that despite a direct effect of body size prior to age 11 years was found, most of the effect of body size during adolescent on adult SBP was mediated through adult body size [6]. In our study, effect of BMI at earlier ages was not evaluated since participants were enrolled only at 13 years of age. Data from the Northern Finland Birth Cohort 1966 also suggested that the effect of BMI 11-15 years was mediated by adult BMI [30], however their findings are not directly comparable with ours since they used BMI growth velocities. Comparison with additional studies assessing the effect of BMI or body weight in childhood or adolescence on later SBP is limited $[5,9,31,32]$, since other statistical methods were applied and direct and indirect effects through adult BMI were not assessed.

The stronger effect of adiposity on insulin resistance is supported by other studies, which, despite methodological differences, found results in line with ours. Longitudinal studies have shown a stronger association of adiposity with insulin resistance than with blood pressure, both in paediatric age [33] and in adulthood [34]. The stronger association of adult adiposity with insulin resistance, and the additional direct effect from adolescent adiposity, in contrast to the completely mediated effect by adult adiposity on blood pressure suggest that the mechanisms involved for blood pressure may be primarily immediate. Mechanisms linking obesity to insulin resistance and blood pressure are complex and not completely understood yet, and therefore further studies are needed to clarify these differences on the outcomes.

Central adiposity (particularly visceral) has been described to be more relevant for cardio metabolic outcomes than total adiposity [17]. In our study, central adiposity was more strongly associated with SBP, in comparison to total adiposity, but not with insulin resistance. Some studies in adolescents have also shown similar effects of total and central adiposity on cardio metabolic outcomes [33,35,36]. Additionally, the WHR in adolescents may not be as good as in adults to represent body fat distribution, and particularly to distinguish visceral from subcutaneous central fat.

In the present study, the results from the life course path analysis suggest that the effect of adolescent adiposity on adult blood pressure and insulin resistance is mostly mediated by adult adiposity. For insulin resistance, there was also an additional direct effect from total adiposity at younger ages. Interventions to reduce adiposity at young ages are expected to result in lower blood pressure and insulin resistance in young adulthood, with greater benefits for insulin resistance.

\section{Conflict of interest}

None. 


\section{Acknowledgements}

This study was supported by national funding from the Portuguese Foundation for Science and Technology (FCT) within the Epidemiology Research Unit - Institute of Public Health, University of Porto (UID/DTP/047507/2013). Individual grants to JA (SFRH/BD/78153/2011) and to SS (SFRH/BD/81123/2011) by the Portuguese Foundation for Science and Technology - FCT are gratefully acknowledged.

\section{Appendix A. Supplementary data}

Supplementary data related to this article can be found at http://dx.doi.org/10.1016/j.numecd.2016.12.007.

\section{References}

[1] McCrindle BW. Will childhood obesity lead to an epidemic of premature cardiovascular disease? Evid Based Cardiovasc Med 2006;10:71-4.

[2] Power C, Thomas C. Changes in BMI, duration of overweight and obesity, and glucose metabolism: 45 years of follow-up of a birth cohort. Diabetes Care 2011;34:1986-91.

[3] Black E, Holst C, Astrup A, Toubro S, Echwald S, Pedersen O, et al. Long-term influences of body-weight changes, independent of the attained weight, on risk of impaired glucose tolerance and Type 2 diabetes. Diabet Med 2005;22:1199-205.

[4] Williams S, Poulton R. Birth size, growth, and blood pressure between the ages of 7 and 26 years: failure to support the fetal origins hypothesis. Am J Epidemiol 2002;155:849-52.

[5] Li L, Law C, Power C. Body mass index throughout the life-course and blood pressure in mid-adult life: a birth cohort study. J Hypertens 2007;25:1215-23.

[6] Gamborg M, Andersen PK, Baker JL, Budtz-Jorgensen E, Jorgensen $T$, Jensen $G$, et al. Life course path analysis of birth weight, childhood growth, and adult systolic blood pressure. Am J Epidemiol 2009;169:1167-78.

[7] Singh AS, Mulder C, Twisk JW, van Mechelen W, Chinapaw MJ. Tracking of childhood overweight into adulthood: a systematic review of the literature. Obes Rev 2008;9:474-88.

[8] Pinto Pereira SM, Power C. Life course body mass index, birthweight and lipid levels in mid-adulthood: a nationwide birth cohort study. Eur Heart J 2013;34:1215-24.

[9] Hardy R, Wadsworth ME, Langenberg C, Kuh D. Birthweight, childhood growth, and blood pressure at 43 years in a British birth cohort. Int J Epidemiol 2004;33:121-9.

[10] De Stavola BL, Nitsch D, dos Santos Silva I, McCormack V, Hardy R, Mann V, et al. Statistical issues in life course epidemiology. Am J Epidemiol 2006;163:84-96.

[11] Lucas A, Fewtrell MS, Cole TJ. Fetal origins of adult disease-the hypothesis revisited. BMJ (Clinical research ed) 1999;319:245-9.

[12] Tu YK, Tilling K, Sterne JA, Gilthorpe MS. A critical evaluation of statistical approaches to examining the role of growth trajectories in the developmental origins of health and disease. Int J Epidemiol 2013;42:1327-39.

[13] Zhou MS, Wang A, Yu H. Link between insulin resistance and hypertension: what is the evidence from evolutionary biology? Diabetol Metab Syndr 2014;6:12.

[14] Dietz WH. Health consequences of obesity in youth: childhood predictors of adult disease. Pediatrics 1998;101:518-25.

[15] Eisenmann JC. Secular trends in variables associated with the metabolic syndrome of North American children and adolescents: a review and synthesis. Am J Hum Biol 2003;15:786-94.

[16] Jiang X, Srinivasan SR, Bao W, Berenson GS. Association of fasting insulin with longitudinal changes in blood pressure in children and adolescents. The Bogalusa Heart Study. Am J Hypertens 1993; 6:564-9.

[17] Britton KA, Massaro JM, Murabito JM, Kreger BE, Hoffmann U, Fox CS. Body fat distribution, incident cardiovascular disease, cancer, and all-cause mortality. J Am Coll Cardiol 2013;62:921-5.

[18] Ramos E, Barros H. Family and school determinants of overweight in 13-year-old Portuguese adolescents. Acta Paediatrica (Oslo, Norway:1992) 2007;96:281-6.

[19] de Onis M, Onyango AW, Borghi E, Siyam A, Nishida C, Siekmann J. Development of a WHO growth reference for school-aged children and adolescents. Bull World Health Organ 2007;85:660-7.

[20] Matthews DR, Hosker JP, Rudenski AS, Naylor BA, Treacher DF Turner RC. Homeostasis model assessment: insulin resistance and beta-cell function from fasting plasma glucose and insulin concentrations in man. Diabetologia 1985;28:412-9.

[21] Lt Hu, Bentler PM. Cutoff criteria for fit indexes in covariance structure analysis: conventional criteria versus new alternatives. Struct Equ Model a Multidiscip J 1999;6:1-55.

[22] Suchanek P, Kralova Lesna I, Mengerova O, Mrazkova J, Lanska V, Stavek P. Which index best correlates with body fat mass BAI, BMI waist or WHR? Neuro Endocrinol Lett 2012;33(Suppl. 2):78-82.

[23] Despres JP. Body fat distribution and risk of cardiovascular disease: an update. Circulation 2012;126:1301-13.

[24] World Health Organization. Waist circumference and waist-hip ratio: report of a WHO expert consultation. Geneva: WHO; 2011.

[25] Lee CM, Huxley RR, Wildman RP, Woodward M. Indices of abdominal obesity are better discriminators of cardiovascular risk factors than BMI: a meta-analysis. J Clin Epidemiol 2008;61: 646-53.

[26] van Dijk SB, Takken T, Prinsen EC, Wittink H. Different anthropometric adiposity measures and their association with cardiovascular disease risk factors: a meta-analysis. Neth Heart J 2012;20: 208-18.

[27] Topouchian J, Agnoletti D, Blacher J, Youssef A, Chahine MN, Ibanez I, et al. Validation of four devices: Omron M6 Comfort, Omron HEM-7420, Withings BP-800, and Polygreen KP-7670 for home blood pressure measurement according to the European Society of hypertension International Protocol. Vasc Health Risk Manag 2014;10:33-44.

[28] Altunkan S, Ilman N, Kayaturk N, Altunkan E. Validation of the Omron M6 (HEM-7001-E) upper-arm blood pressure measuring device according to the International Protocol in adults and obese adults. Blood Press Monit 2007;12:219-25.

[29] Tirosh A, Shai I, Afek A, Dubnov-Raz G, Ayalon N, Gordon B, et al. Adolescent BMI trajectory and risk of diabetes versus coronary disease. N Engl J Med 2011;364:1315-25.

[30] Kaakinen M, Sovio U, Hartikainen AL, Pouta A, Savolainen MJ, Herzig $\mathrm{KH}$, et al. Life course structural equation model of the effects of prenatal and postnatal growth on adult blood pressure. J Epidemiol Community Health 2014;68:1161-7.

[31] Eriksson J, Forsen T, Tuomilehto J, Osmond C, Barker D. Fetal and childhood growth and hypertension in adult life. Hypertension 2000;36:790-4.

[32] Law CM, Shiell AW, Newsome CA, Syddall HE, Shinebourne EA, Fayers PM, et al. Fetal, infant, and childhood growth and adult blood pressure: a longitudinal study from birth to 22 years of age. Circulation 2002;105:1088-92.

[33] Lawlor DA, Benfield L, Logue J, Tilling K, Howe LD, Fraser A, et al. Association between general and central adiposity in childhood, and change in these, with cardiovascular risk factors in adolescence: prospective cohort study. BMJ (Clinical research ed) 2010; 341. c6224.

[34] Juonala M, Magnussen CG, Berenson GS, Venn A, Burns TL, Sabin MA, et al. Childhood adiposity, adult adiposity, and cardiovascular risk factors. N Engl J Med 2011;365:1876-85.

[35] Graves L, Garnett SP, Cowell CT, Baur LA, Ness A, Sattar N, et al. Waist-to-height ratio and cardiometabolic risk factors in adolescence: findings from a prospective birth cohort. Pediatr Obes 2014;9:327-38.

[36] Adegboye AR, Andersen LB, Froberg K, Sardinha LB, Heitmann BL. Linking definition of childhood and adolescent obesity to current health outcomes. Int J Pediatr Obes 2010;5:130-42. 